\title{
Epigenetic alterations are involved in the overexpression of glutathione $S$-transferase $\pi$-1 in human colorectal cancers
}

\author{
RUI ZHANG ${ }^{1,2}$, KYOUNG AH KANG $^{1}$, MEI JING PIAO ${ }^{1}$, KI CHEON KIM $^{1}$, JIAN ZHENG $^{1}$, \\ CHENG WEN YAO $^{1}$, JI WON CHA ${ }^{1}$, YOUNG HEE MAENG ${ }^{1}$, WEON YOUNG CHANG ${ }^{1}$, \\ PYONG-GON MOON ${ }^{3}$, MOON-CHANG BAEK ${ }^{3}$ and JIN WON HYUN ${ }^{1}$ \\ ${ }^{1}$ School of Medicine and Institute for Nuclear Science and Technology, Jeju National University, \\ Jeju 690-756, Republic of Korea; ${ }^{2}$ School of Medical Science and Laboratory Medicine, Jiangsu University, \\ Jiangsu 212013, P.R. China; ${ }^{3}$ Department of Molecular Medicine, Cell and Matrix Biology Research Institute, \\ School of Medicine, Kyungpook National University, Daegu 700-422, Republic of Korea
}

Received April 18, 2014; Accepted June 6, 2014

DOI: 10.3892/ijo.2014.2522

\begin{abstract}
Glutathione S-transferase $\pi-1$ (GSTP-1) is a member of the glutathione S-transferase enzyme superfamily, which catalyzes the conjugation of electrophiles to glutathione during the process of detoxification. In this study, the epigenetic alterations of GSTP-1 expression in human colorectal cancers and the underlying mechanisms were investigated. In 10 colon cancer patients, proteomic analysis revealed that expression of GSTP-1 protein was higher in tumor tissues than in paired adjacent normal tissues. Likewise, in 7 of 10 colon cancer patients, GSTP-1 protein expression was more than 1.5-fold higher in tumor tissues than in adjacent normal tissues, as determined by western blotting. Immunohistochemical data confirmed that GSTP-1 protein was expressed at higher levels in colon cancer tissues compared to normal mucosa. GSTP-1 enzyme activity was closely correlated with GSTP-1 protein expression in colon cancer patients. Consistent with this, GSTP-1 mRNA, protein and activity levels were higher in the colorectal cancer cell lines Caco-2, HCT-116, HT-29, SNU-407 and SNU-1033 compared to the normal colon cell line FHC. Methylation-specific PCR results indicated that the high levels of GSTP-1 in human colorectal cancer cell lines were likely due to the lower degree of promoter methylation in colon cancer cell lines compared to the normal colon cell line, consistent with findings in colon cancer patients. Moreover, the levels of specific activatorprotein complexes and histone marks were higher in human colorectal cancer cells compared to the normal human colon cell line, whereas the repressor protein complexes exhibited the opposite pattern. Furthermore, chromatin immunoprecipitation
\end{abstract}

Correspondence to: Professor Jin Won Hyun, School of Medicine, Jeju National University, Jeju 690-756, Republic of Korea

E-mail: jinwonh@jejunu.ac.kr

Key words: glutathione S-transferase $\pi-1$, epigenetic alteration, colorectal cancer assays demonstrated that expression levels of the transcription factors AP-1 and SP-1 were correlated with the upregulation of GSTP-1 expression in colorectal cancer cells. Finally, knockdown of GSTP-1 promoted the sensitivity of SNU-407 cells to the anticancer agent 5-fluorouracil. These data indicate that GSTP-1 may serve as a clinically useful biomarker of colon cancer and a target for anti-colon cancer drugs.

\section{Introduction}

Glutathione S-transferases (GSTs) are phase-II detoxification enzymes that catalyze the conjugation of glutathione to the electrophilic centers of multiple types of endogenous and exogenous electrophiles, including genotoxins, carcinogens and anticancer agents $(1,2)$. Based on their biochemical properties, the cytosolic GSTs are divided into the following subclasses: alpha $(\alpha)$, mu $(\mu)$, omega $(\omega)$, pi $(\pi)$, sigma $(\sigma)$, theta $(\theta)$ and zeta $(\zeta)$. GST pi-1 (GSTP-1) is encoded by a single gene mapped to chromosome $11 \mathrm{q} 13(3,4)$ and is frequently overexpressed in many types of tumors, including colorectal cancers $(5,6)$. In addition to its enzymatic functions, GSTP-1 is also thought to possess non-enzymatic functions. For example, GSTP-1 acts as a regulator of cell signaling in apoptosis through its interactions with other proteins, such as c-Jun NH2-terminal kinase $(7,8)$. These enzymatic and non-enzymatic functions of GSTP-1 are correlated with drug resistance, failure of therapy and poor survival in patients with tumors expressing high levels of GSTP-1 $(8,9)$.

Currently, the role of GSTP-1 in carcinogenesis is controversial. Loss of GSTP-1 function is associated with multiple types of cancers, corroborating the notion that GSTP-1 may be a tumor suppressor. For example, mice lacking GSTP-1 exhibit increased skin tumorigenesis (10) and it has been proposed that hypermethylated GSTP-1 represents a potential epigenetic biomarker for prostate cancer (11). By contrast, overexpression of GSTP-1 is associated with the development of other types of cancer as well as with the acquisition of drug resistance in some neoplasms $(12,13)$, suggesting that GSTP-1 can also act as an oncogene. In particular, GSTP-1 is overexpressed 
in all stages of colorectal cancer, from aberrant crypt foci to advanced carcinomas $(5,6)$. Colorectal cancer is the third most commonly diagnosed cancer in the world, and an increased incidence of colon cancer has been reported in recent years in Asian countries including Korea (14). Colorectal cancer develops through multiple steps that involve sequential acquisition of genetic alterations in key tumor suppressors and oncogenes (15). To attempt to resolve the controversy regarding the role of GSTP-1, we investigated the epigenetic alteration of GSTP-1 in human colorectal cancers and explored the underlying mechanisms in detail.

The two major epigenetic controls involved in transcriptional silencing are covalent modification of histones and DNA CpG-island methylation (16). Post-translational modifications of histones include acetylation, methylation, phosphorylation and ubiquitination. Each of these modifications recruits regulatory factors that influence chromatin structure and transcription. Reversible histone acetylation regulates nucleosome-nucleosome and nucleosome-DNA interactions, which in turn influence the access of transcriptional factors to DNA (17). The balance of acetylation is regulated by two types of enzymes with opposing activities, acetyltransferases and deacetylases (18). DNA methylation is responsible for transcriptional regulation and has been implicated in transcriptional silencing (19). Hypermethylation of a promoter region leads to the formation of a repressor complex composed of methyl-CpG-binding proteins (MBDs), DNA methyltransferases (DNMTs), and histone deacetylases (HDACs) $(20,21)$. This DNA-protein complex induces histone deacetylation and catalyzes the methylation of lysine 9 and 27 in histone H3, compacting the structural arrangement of nucleosomes and thereby generating heterochromatin $(22,23)$.

By contrast, the absence of methylated CpG sites, DNMTs, MBDs and HDACs from gene promoters is a hallmark of transcriptionally active euchromatin. Abnormal gene-specific demethylation can lead to overexpression of genes that contribute to disease (24). Additionally, modifications of lysine 4 in histone $\mathrm{H} 3$, such as trimethylation ( $\mathrm{H} 3 \mathrm{~K} 4 \mathrm{me} 3)$, are also associated with transcriptionally permissive chromatin $(25,26)$. Analysis of the human GSTP-1 proximal promoter region revealed the presence of putative binding sites for SP-1, AP-1 and NF- $\kappa \mathrm{B}$, indicating that these transcription factors play important roles in the regulation of GSTP-1 expression (27-29).

In this study, we tested the hypothesis that overexpression of GSTP-1 in human colorectal cancers is partially due to the lower degree of DNA methylation and histone modification in tumor cells relative to normal colon cells and tissues, and that transcription factors are involved in this regulation.

\section{Materials and methods}

Materials. Primary mouse monoclonal anti-GSTP-1, antiDNMT1, anti-MBD2 antibodies and primary rabbit polyclonal anti-histone H3 (tri-methyl K4) and anti-histone H3 (tri-methyl K9) antibodies were purchased from Abcam, Inc. (Cambridge, MA, USA). Primary rabbit anti-acetyl-histone H3 (K9) and anti-c-Jun antibodies were purchased from Cell Signaling Technology, Inc. (Beverly, MA, USA). Primary mouse monoclonal anti-HDAC1 antibody was purchased from Santa Cruz Biotechnology (Santa Cruz, CA, USA). [3-(4,5-Dimethyl- thiazol-2-yl)-2,5-diphenyltetrazolium] bromide (MTT) and primary rabbit anti- $\beta$-actin antibody were purchased from Sigma-Aldrich Chemical Co. (St. Louis, MO, USA). All other chemicals and reagents were of analytical grade.

Patient tissues. The biospecimens and data used in this study were provided by the Biobank of Jeju National University Hospital, a member of Korea Biobank Network (Jeju, Korea) (A03-2). This study was approved by the institutional review board for ethics of Jeju National University Hospital (IRB 2011-38), and informed written consent was obtained from all the patients.

Cell culture. The human colon cancer cell lines Caco-2, HCT-116, HT-29, SNU-407 and SNU-1033 were obtained from the Korean Cell Line Bank (Seoul, Korea), and the normal human colon cell line FHC was purchased from the American Type Culture Collection (Rockville, MD, USA). Cells were maintained at $37^{\circ} \mathrm{C}$ in an incubator with a humidified atmosphere containing $5 \% \mathrm{CO}_{2}$. HCT-116, HT-29, SNU-407 and SNU-1033 cells were cultured in RPMI-1640 medium containing $10 \%$ heat-inactivated fetal bovine serum (FBS), streptomycin $(100 \mu \mathrm{g} / \mathrm{ml})$ and penicillin $(100 \mathrm{U} / \mathrm{ml})$. Caco-2 cells were cultured in MEM medium containing $10 \%$ heat-inactivated FBS, streptomycin $(100 \mu \mathrm{g} / \mathrm{ml})$ and penicillin $(100 \mathrm{U} / \mathrm{ml})$. FHC cells were cultured in a 1:1 mixture of Ham's F12 and DMEM containing HEPES (25 mM), cholera toxin (10 ng/ml; Calbiochem-Novabiochem Corp., La Jolla, CA, USA), insulin $(5 \mu \mathrm{g} / \mathrm{ml})$, transferrin $(5 \mu \mathrm{g} / \mathrm{ml})$, hydrocortisone $(100 \mathrm{ng} / \mathrm{ml})$ and $10 \% \mathrm{FBS}$.

Proteomic analysis. Two samples were pooled from 10 colon cancer and normal tissue, respectively. The extracted proteins from tissues were digested using modified gel-assisted digestion protocol (30). The gel pieces containing proteins were reduced and alkylated. Proteolytic digestion was conducted with $100 \mathrm{ng} / \mu \mathrm{l}$ trypsin and incubated at $37^{\circ} \mathrm{C}$ overnight. The peptides were extracted from the gel. The nanoscale LC separation of tryptic peptides was performed by using a nanoAcquity system (Waters Corp., Milford, MA, USA), equipped with a Symmetry C18 $5 \mu \mathrm{m}, 5 \mathrm{~mm} \times 300 \mu \mathrm{m}$ precolumn and an $\mathrm{BEH}$ C18 $1.7 \mu \mathrm{m}, 25 \mathrm{~cm} \times 75 \mu \mathrm{m}$ analytical reversed phase column (Waters Corp.). The samples were initially transferred, with an aqueous $0.1 \%$ formic acid solution, to the precolumn at a flow rate of $10 \mu \mathrm{l} / \mathrm{min}$ for $3 \mathrm{~min}$. The mobile phase A was composed of water with $0.1 \%$ formic acid, and the mobile phase B was composed of $0.1 \%$ formic acid in acetonitrile. The peptides were separated with a gradient of $3-40 \%$ mobile phase B over $180 \mathrm{~min}$ at a flow rate of $300 \mathrm{nl} / \mathrm{min}$, followed by a $25 \mathrm{~min}$ rinse with $90 \%$ of mobile phase B. The analysis of tryptic peptides was performed using a Q-Tof Premier mass spectrometer (Waters Corp.). Tryptic peptide was detected within the retention time range of approximately $0.12 \mathrm{~min}$ and at mass precision of approximately $3.1 \mathrm{ppm}$ from three independent runs. Accurate mass LC-MS data were collected in an alternating, low energy and elevated energy mode of acquisition (31). Continuum LC-MS data were processed for generating peaklist and searched, using ProteinLynx GlobalServer v2.3 IdentityE (Waters Corp.). All MS/MS data were searched against the IPI human protein database (version 3.36; 
containing 69,012 sequences and 29,002,682 residues) using the Proteinlynx Global Server v2.3 IdentityE software. All database searches allowed for fixed modification in regard to the cysteine residue and variable modification in regard to the methionine. The relative quantitation was performed using the precursor intensity measurements available in the clustered exact mass and retention time. The redundant quantitative measurements provided by the multiple tryptic peptides from each protein were used to determine an average relative fold-change. A 95\% confidence interval was determined for each average fold-change from the standard deviation of the observed quantitative measurements and the total number of observed tryptic peptides.

Western blot analysis. Cells were lysed on ice for $30 \mathrm{~min}$ in $100 \mu \mathrm{l}$ lysis buffer [ $120 \mathrm{mM} \mathrm{NaCl}, 40 \mathrm{mM}$ Tris (pH 8.0), $0.1 \%$ NP-40] and centrifuged at 13,000 x g for $15 \mathrm{~min}$. Supernatants were collected and the protein concentrations were determined. Aliquots containing $40 \mu \mathrm{g}$ of protein were boiled for $5 \mathrm{~min}$ and electrophoresed on $10 \%$ SDS-polyacrylamide gels. Proteins were transferred onto nitrocellulose membranes, which were subsequently incubated with anti-GSTP-1 antibody overnight at $4^{\circ} \mathrm{C}$. The membranes were further incubated with secondary horseradish peroxidase-conjugated anti-immunoglobulin- $\mathrm{G}$ (Pierce, Rockford, IL, USA). Protein bands were detected using an enhanced chemiluminescence western blotting detection kit (Amersham, Little Chalfont, Buckinghamshire, UK).

Immunohistochemistry for detection of GSTP-1. For detection of GSTP1 in tissue specimens, tissue specimens were fixed in $10 \%$ buffered formalin and embedded in paraffin. The same paraffin-embedded tissues as those used for the original hematoxylin and eosin stained sections were used for immunohistochemistry. Tissue blocks were cut into $3 \mu \mathrm{m}$ thick slices and mounted on SuperFrost ${ }^{\circledR}$ Plus coated slides. Sections were then deparaffinized in xylene and rehydrated through a graded ethanol series. A standard immunohistochemical technique was performed using a Ventana BenchMark XT immunostainer with the anti-GSTP-1 antibody at a dilution of 1:100. Antigen retrieval on the immunostainer was carried out for $30 \mathrm{~min}$. The anti-GSTP-1 antibody was incubated at $37^{\circ} \mathrm{C}$ for $60 \mathrm{~min}$, and 3,3'-diaminobenzidine was used as a chromogen; slides were counterstained with hematoxylin prior to mounting. All staining procedures were performed according to the manufacturer's recommendations. Intramucosal mononuclear cells in the tissue samples, which stain strongly, served as internal positive controls. Negative controls for non-specific binding were obtained by omitting the primary antibody. The immunohistochemical slides were evaluated and interpreted by a pathologist. For detection of GSTP-1 in cells, cells plated on coverslips were fixed with $4 \%$ paraformaldehyde for $30 \mathrm{~min}$ and permeabilized with PBS containing $0.1 \%$ Triton X-100 for $2.5 \mathrm{~min}$. Cells were then treated with blocking solution (PBS containing 3\% bovine serum albumin) for $1 \mathrm{~h}$, and then incubated for $2 \mathrm{~h}$ with anti-GSTP-1 antibody diluted in blocking solution. Immunoreacted primary anti-GSTP-1 antibody was detected by incubating samples for $1 \mathrm{~h}$ with a FITC-conjugated secondary antibody at a dilution of 1:200 (Jackson Immuno Research Laboratories, West Grove, PA, USA). After washing with PBS, stained cells were mounted onto microscope slides in mounting medium. Microscopic images were collected on a confocal microscope using the Laser Scanning Microscope 5 PASCAL software (Carl Zeiss, Jena, Germany).

Assessment of GST activity. GST enzyme activity in colon tissues or cells was measured using the GST activity assay kit (Abcam, Inc.). The GST substrate 1-chloro-2,4-dinitrobenzene (CDNB) was used to assess GST-related enzyme activity. Briefly, colon tissues or cells were homogenized in $0.5 \mathrm{ml} \mathrm{GST}$ assay buffer, and homogenates were centrifuged at $10,000 \mathrm{x} \mathrm{g}$ for $15 \mathrm{~min}$ at $4^{\circ} \mathrm{C}$. The supernatants were collected and the protein concentrations were determined. Samples were prepared with GST assay buffer in a total volume of $50 \mu \mathrm{l}$, including a negative control (50 $\mu \mathrm{l}$ GST assay buffer alone) and a positive control (10 $\mu \mathrm{l}$ of GST positive control diluted 1:50 and $40 \mu \mathrm{l}$ GST assay buffer). To each well containing sample or control, $5 \mu \mathrm{l}$ of glutathione was added, followed by $50 \mu \mathrm{l}$ of substrate mixture (45 $\mu \mathrm{l}$ of GST assay buffer and $5 \mu \mathrm{l}$ of CDNB). After addition of the substrate mixture, plates were shaken carefully to start the reaction, and the absorbance at $340 \mathrm{~nm}$ was read once every minute to obtain at least 5 time points. The change in absorbance $\left(\Delta \mathrm{A}_{340}\right)$ per minute was determined by plotting the absorbance values as a function of time to obtain the slope of the linear portion of the curve. The reaction rate was then determined using the CDNB extinction coefficient at $340 \mathrm{~nm}$, $0.0053 \mu \mathrm{M}^{-1}$. GST activity is expressed as $\mathrm{nmol} / \mathrm{min} / \mathrm{ml}$.

RNA isolation and reverse transcription-polymerase chain reaction $(R T-P C R)$. Total RNA was isolated from cells using the easy-BLUE ${ }^{\mathrm{TM}}$ total RNA extraction kit (iNtRON Biotechnology, Inc., Korea), and cDNA was amplified using $1 \mu 1$ of the reverse transcription reaction buffer, primers, dNTPs and $0.5 \mathrm{U}$ of Taq DNA polymerase in a final volume of $25 \mu \mathrm{l}$. The PCR conditions were: initial denaturation for $5 \mathrm{~min}$ at $94^{\circ} \mathrm{C} ; 35$ cycles of $94^{\circ} \mathrm{C}$ for $30 \mathrm{sec}, 60^{\circ} \mathrm{C}$ for $30 \mathrm{sec}$, and $72^{\circ} \mathrm{C}$ for $30 \mathrm{sec}$; and a final elongation step at $72^{\circ} \mathrm{C}$ for $7 \mathrm{~min}$. The following primers were used to amplify GSTP1 cDNA: forward, 5'-ATGCCGCCCTACACCGTG-3'; reverse, 5'-CCAGGTGACGCAGGATGG-3'. The amplified products were resolved by electrophoresis on a $1 \%$ agarose gel, stained with RedSafe ${ }^{\mathrm{TM}}$ nucleic acid staining solution (iNtRON Biotechnology, Inc.) and photographed under UV light using the Image Quant ${ }^{\mathrm{TM}}$ TL analysis software (Amersham Bioscience, Sweden).

Methylation-specific (MS)-PCR. DNA bisulfate modification was carried out using the Methylamp ${ }^{\mathrm{TM}}$ DNA modification kit (Epigentek, Pittsburgh,PA,USA), according to the manufacturer's instructions. To analyze GSTP-1 DNA methylation, MS-PCR was performed using an EpiTect MSP kit (Qiagen, Valencia, CA, USA). Primers to perform MS-PCR on the GSTP-1 promoter were designed using the Methyl Primer Express ${ }^{\circledR}$ software v1.0 (Applied Biosystems). The methylated and unmethylated GSTP1 primer sets used for PCR were as follows: unmethylated GSTP-1 forward, 5'-GGAGTTTTGTTGTTGTAGTTTTT-3', and reverse, 5'-CCACTAACAACACTAAAAACATC-3'; methylated GSTP-1 forward, 5'-GTTTCGTCGTCGTAGTTTTC-3', and reverse, 5'-CCGCTAACGACACTAAAAAC-3'. The amplified products were resolved by electrophoresis on a $3 \%$ agarose gel, stained with RedSafe nucleic acid staining solution 
A

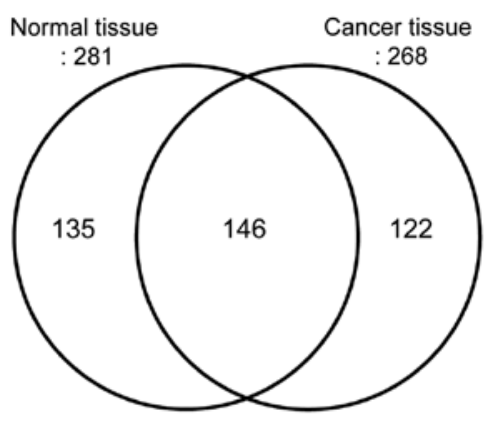

B

\begin{tabular}{c|c|c}
\hline Name & Score & Cancer: Normal Ratio \\
\hline PDCD6IP protein & 1290.21 & 0.8 \\
Dopamine receptor interacting protein 4 & 1273.91 & 0.8 \\
Glutathione S-transferase & 1129.04 & 1.5 \\
P-glycoprotein 1 & 1068.01 & Normal only \\
Multidrug resistance protein 1 & 1061.79 & Normal only \\
ubiquitin C & 896.34 & 1.0 \\
Hemoglobin subunit beta & 805.6 & Cancer only \\
BRO1 domain-containing protein BROX & 781.93 & 1.3 \\
MYO1C & 724.95 & Normal only \\
Serum albumin precursor & 724.94 & 2.0 \\
Glutamyl aminopeptidase & 706.15 & Normal only \\
Dipeptidase 1 precursor & 693.63 & Normal only \\
Ezrin & 684.39 & 1.1 \\
Keratin, type II cytoskeletal 1 & 666.84 & 0.7 \\
Olfactomedin-4 precursor & 650.08 & 1.8 \\
Prominin-1 precursor & 609.9 & 0.2 \\
Calpain-7 & 607.9 & Cancer only \\
Heat shock cognate 71 kDa protein & 599.87 & 1.1 \\
Aminopeptidase N & 598.4 & 0.5 \\
Pro-epidermal growth factor precursor & 584.98 & 1.2 \\
\hline \multicolumn{2}{c}{} &
\end{tabular}

Figure 1. Proteomic analysis from colon cancer and normal tissue. (A) Venn diagram of overlapping indicates proteins between colon cancer and normal tissue. (B) Significant proteins are listed using PLGS scores from colon cancer compared to normal tissue.

and photographed under UV light using Image Quant ${ }^{\mathrm{TM}}$ TL analysis software.

Chromatin immunoprecipitation (ChIP) assay. Cells were processed using the Simple ChIP ${ }^{\mathrm{TM}}$ enzymatic chromatin IP kit (Cell Signaling Technology, Inc.), according to the manufacturer's instructions. Briefly, the procedure was initiated by cross-linking the proteins to DNA by adding $1 \%$ formaldehyde to the culture dishes and rocking on a platform for $10 \mathrm{~min}$ at room temperature. The cross-linking was stopped by the addition of glycine solution. Cells were washed twice with ice-cold PBS, pelleted by centrifugation and resuspended in $1 \mathrm{ml}$ cell lysis buffer containing protease inhibitor. The soluble chromatin was sheared by sonication followed by centrifugation at $15,000 \mathrm{x}$ g. Diluted supernatants were pre-cleared and blocked with protein $\mathrm{A} / \mathrm{G}$ agarose, and the sonicated chromatin-DNA complex was precipitated overnight with the antibodies of interest. Bound DNA was eluted by incubating the beads in elution buffer, purified and amplified using primers flanking the SP-1 and AP-1 binding sites within the human GSTP1 proximal promoter. The oligonucleotide containing the transcription factor binding site in the GSTP-1 promoter was obtained from Bioneer (Seoul, Korea). The ChIP procedure was analyzed by PCR using human GSTP-1 promoter-specific primers as follows (32): forward, 5'-GCAG CGGTCTTAGGGAATTT-3'; reverse, 5'-CTTTCCCTCTTT CCCAGGTC-3'. The cycle parameters were as follows: $95^{\circ} \mathrm{C}$ for $5 \mathrm{~min} ; 40$ cycles of $95^{\circ} \mathrm{C}$ for $30 \mathrm{sec}, 60^{\circ} \mathrm{C}$ for $30 \mathrm{sec}$ and $72^{\circ} \mathrm{C}$ for $30 \mathrm{sec}$; and a final extension at $72^{\circ} \mathrm{C}$ for $7 \mathrm{~min}$. The amplified products were resolved by electrophoresis on a $3 \%$ agarose gel, stained with RedSafe nucleic acid staining solution and photographed under UV light using Image Quant ${ }^{\mathrm{TM}}$ TL analysis software.

Transient transfection of small interfering RNA (siRNA). SNU-407 cells were seeded at a density of $1.5 \times 10^{5}$ cells/well in 24-well plates and allowed to reach approximately 50\% confluency on the day of transfection. The siRNA constructs were mismatched siRNA Control and siRNA GSTP-1 (Santa Cruz Biotechnology). Cells were transfected with 10-50 nM siRNA using Lipofectamine ${ }^{\mathrm{TM}} 2000$ (Invitrogen) according to the manufacturer's instructions. At $24 \mathrm{~h}$ after transfection, cells were treated with 5-fluorouracil (5-FU) for $48 \mathrm{~h}$, and then examined by the MTT assay.

Cell viability. The effect of GSTP-1 on the viability of human colon cancer cells was determined using the MTT assay, which is based on the reduction of a tetrazolium salt by mitochondrial 

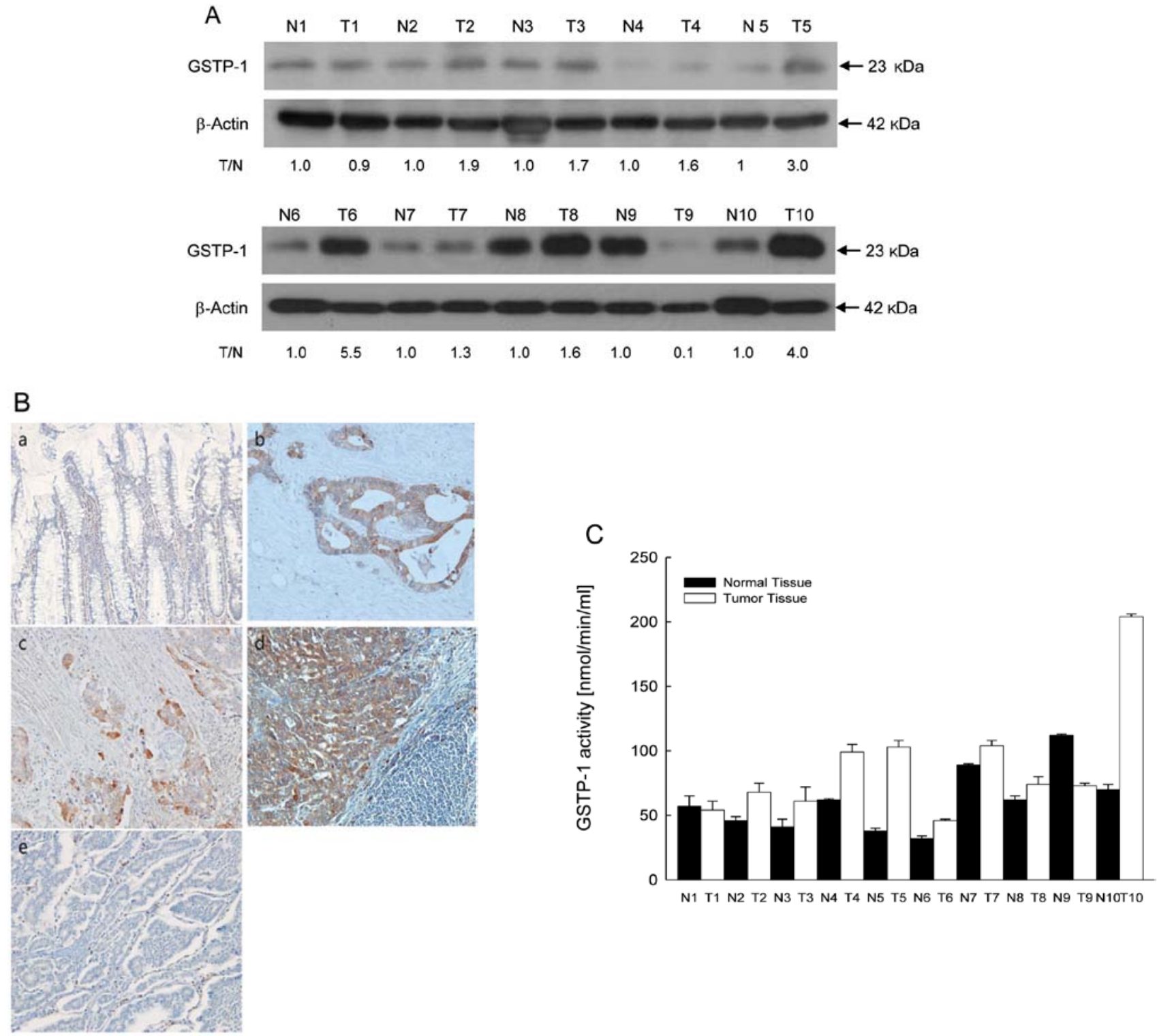

Figure 2. Protein and activity levels of GSTP-1 in colon cancer patients. (A) Expression of the GSTP-1 protein was detected using an anti-GSTP-1 antibody, and band intensities were quantified. (B) Immunohistochemistry for GSTP-1 in colon carcinoma tissues revealed (a) negative staining in normal mucosa; (b) and (c) moderate expression in well and moderately differentiated adenocarcinoma; (d) strong positivity in poorly differentiated adenocarcinoma; and (e) no expression in neuroendocrine carcinoma. (C) GST enzyme activity in colon tissues was measured as described in Materials and methods. GST activity is expressed as nmol/min/ml.

dehydrogenase in viable cells (33). Cells were treated with 5-FU at a concentration of $800 \mu \mathrm{M}$. MTT $(50 \mu \mathrm{l}$ of a $2 \mathrm{mg} / \mathrm{ml}$ stock solution) was added to each well to obtain a total reaction volume of $200 \mu \mathrm{l}$. After incubation for $4 \mathrm{~h}$, the supernatants were aspirated. Formazan crystals present in each well were dissolved in $150 \mu \mathrm{l}$ of dimethyl sulfoxide, and the absorbance at $540 \mathrm{~nm}$ was measured on a scanning multi-well spectrophotometer.

Statistical analysis. The results were subjected to analysis of variance using Tukey's test to analyze differences. $\mathrm{P}<0.05$ was considered statistically significant.

\section{Results}

GSTP-1 protein expression and enzyme activity in normal and colon carcinoma tissues. To compare the fold-changes of expressed proteins from normal and colon carcinoma tissues, shotgun proteomic approach based on label-free quantification was applied. A total of 403 non-redundant proteins were identified from the analysis of normal and colon carcinoma tissues. Among them, 146 proteins were identified in common. The numbers of unique proteins were 135 and 122 proteins in normal and cancer tissue, respectively (Fig. 1A). To show disease-specific proteins, we listed up some proteins based on score of protein recovery quantification which is proportional to accuracy of quantification (Fig. 1B). Among them, we focused on glutathione S-transferase which was overexpressed in the cancer group. To confirm this proteomic result, GSTP-1 protein level and enzyme activity were assessed in colon cancer tissues from 10 patients by western blotting and an enzyme activity assay, respectively. The expression level of GSTP-1 protein that showed $>1.5$-fold higher than in corresponding normal tissues was detected in $70 \%$ (7/10) of tumor tissues from colon cancer patients (Fig. 2A). Immunohistochemical analysis revealed faint expression of 


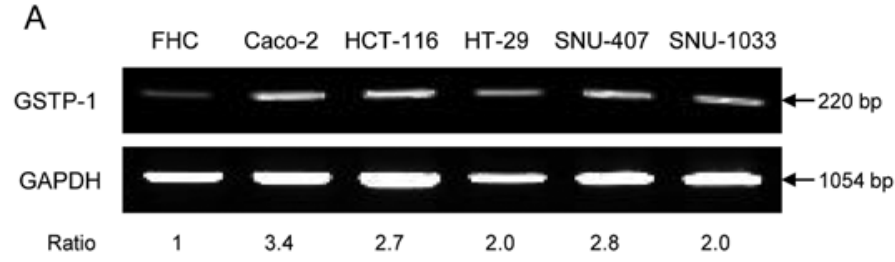

B

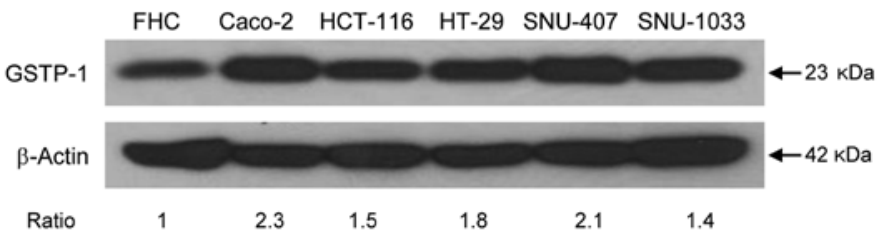

C

HCT-116

HT-29
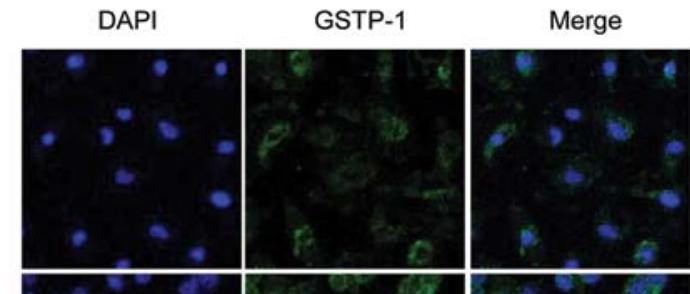

SNU-407
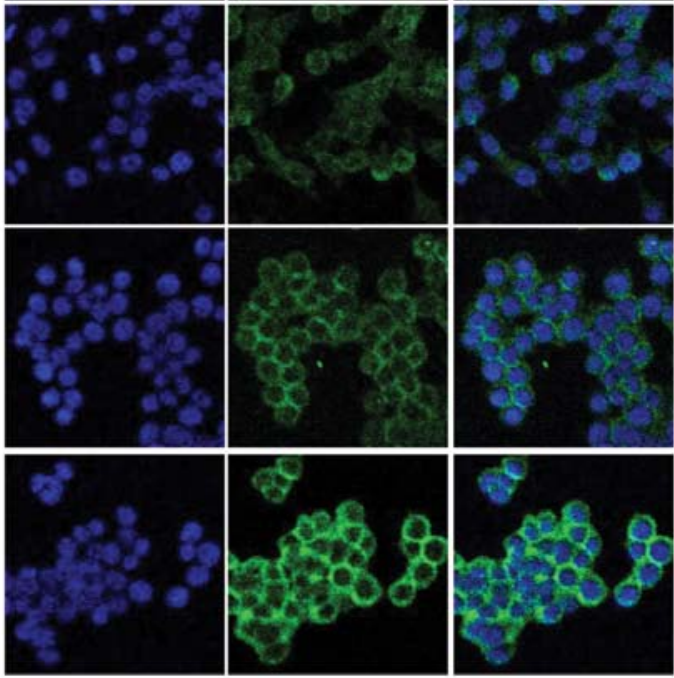

GSTP-1 protein in normal mucosa (Fig. 2Ba). The carcinoma cells exhibited increased GSTP-1 expression in the cytoplasm, which was distributed in a diffuse pattern (Fig. 2Bb-d), and some cells were strongly positive (Fig. 2Bd). Neuroendocrine carcinoma cells in specimens exhibited no GSTP-1 expression (Fig. 2Be). GST enzyme activity was higher in tumor tissues than in normal tissues from colon cancer patients, consistent with the western blotting data (Fig. 2C). These results demonstrate that GSTP-1 is highly expressed in tumor tissues from Korean colon cancer patients, suggesting that this protein might be used as a biomarker for cancer detection.

GSTP-1 mRNA and protein expression and enzyme activity in human colon cancer cell lines. The assessment of GSTP-1 expression in colon cancer cell lines by RT-PCR and western blotting revealed that GSTP-1 mRNA (Fig. 3A) and protein levels (Fig. 3B) were higher in the colon cancer cell lines

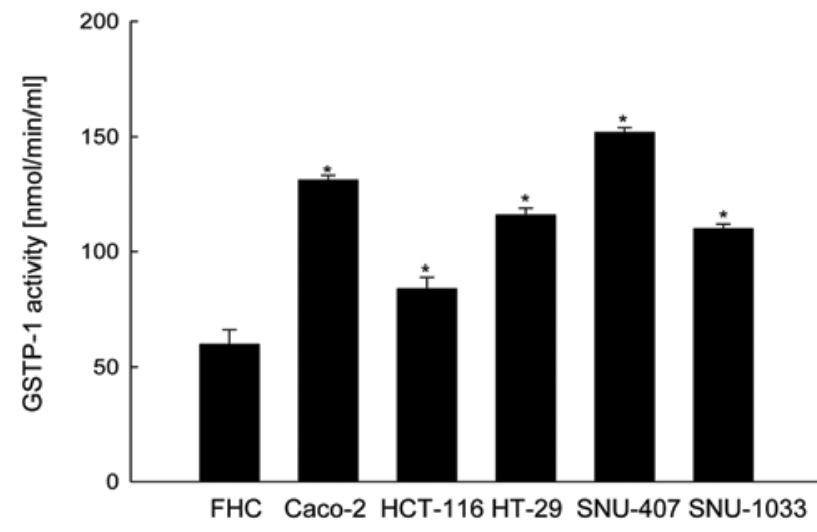

Figure 3. GSTP-1 mRNA, protein, and activity levels in normal colon and cancer cell lines. (A) The GSTP-1 mRNA level was analyzed by RT-PCR and quantified. GSTP-1 protein was detected by (B) western blotting and (C) fluorescence microscopy. (D) GST enzyme activity in colon tissues was measured as described in Materials and methods. GST activity is expressed as $\mathrm{nmol} / \mathrm{min} / \mathrm{ml}$. *Significantly different from FHC cells $(\mathrm{P}<0.05)$.

Caco-2, HCT-116, HT-29, SNU-407 and SNU-1033 than in the normal colon cell line FHC. The protein expression and cytoplasmic localization of GSTP-1 was assessed by confocal microscopy (Fig. 3C), yielding data consistent with the western blotting results. GST enzyme activity was also higher in cancer cell lines, consistent with the pattern of GSTP-1 protein expression (Fig. 3D), although both GSTP-1 expression and activity levels differed among the cell lines.

Methylation status of GSTP-1 regulatory regions in normal and colon carcinoma tissues and human colon cancer cell lines. We next investigated why the expression of GSTP-1 is higher in colorectal cancers than in normal tissues. To this end, the methylation status of GSTP-1 regulatory regions was determined by MS-PCR to elucidate the relationship between methylation status and protein expression. The methylation status of GSTP-1 in patient tissues was determined. While the relationship between methylation degree and protein expression in tissues was not close enough (Fig. 4A). Intriguingly, GSTP-1 was methylated to a lesser extent in the colon cancer cell lines Caco-2, HCT-116, SNU-407 and SNU-1033, but not HT-29 (Fig. 4B), than in the FHC cell line, almost concordant 
A

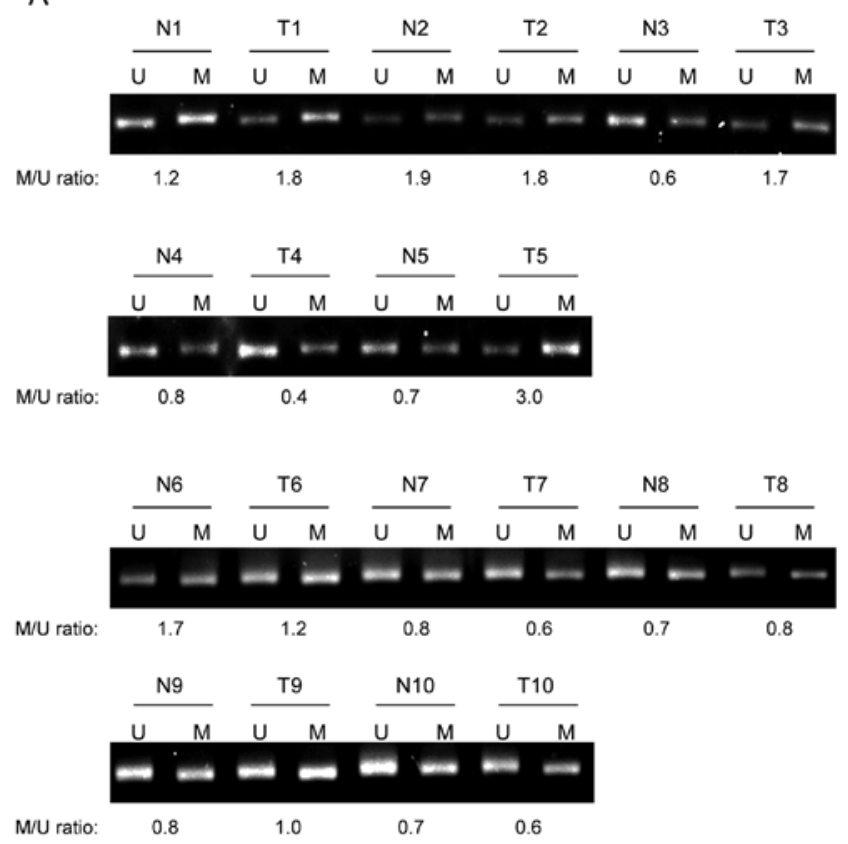

B

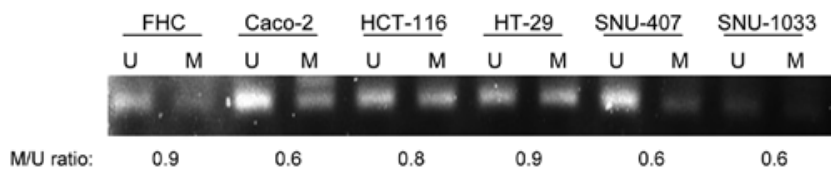

Figure 4. Methylation status of the GSTP-1 promoter region in cancer tissues and human colorectal cancer cell lines. MS-PCR analysis of GSTP-1 was performed using genomic DNA isolated from (A) patient tissues and (B) human colorectal cancer cells. Methylation was determined by detection of a PCR product amplified by methylation-specific primers. Demethylation was detected by the presence of a PCR product amplified by unmethylation-specific primers. U, unmethylated DNA; M, methylated DNA.

with GSTP-1 protein expression. These results suggest that overexpression of GSTP-1 in human colon cancers is only partially due to decreased methylation of the GSTP-1 regulatory region.

Correlation of GSTP-1 transcriptional activity with DNA methylation and histone modification. We next performed ChIP assays to investigate the epigenetic-related proteins associated with the GSTP-1 promoter region. The levels of DNA methylation- and heterochromatin-associated proteins such as DNMT1, MBD2 and HDAC1 were lower in the HCT-116 and SNU-407 cell lines than in the FHC cell line. In addition, the GSTP-1 promoter in HCT-116 and SNU-407 cells was highly enriched for trimethylation of lysine 4 of histone $\mathrm{H} 3$ (H3K4me3) and for acetylation of histone $\mathrm{H} 3$ (AcH3), which induce active transcription (Fig. 5A). Whereas the level of $\mathrm{H} 3 \mathrm{~K} 9 \mathrm{me} 3$, which represses transcription, was lower in two cancer cell lines than in the FHC line (Fig. 5A). The GSTP-1 promoter region contains binding sites for the transcription factors SP-1 and AP-1. We performed ChIP assays to determine the roles of c-Jun (a component of AP-1) and SP-1 in the regulation of GSTP-1 expression. The c-Jun and SP-1 were

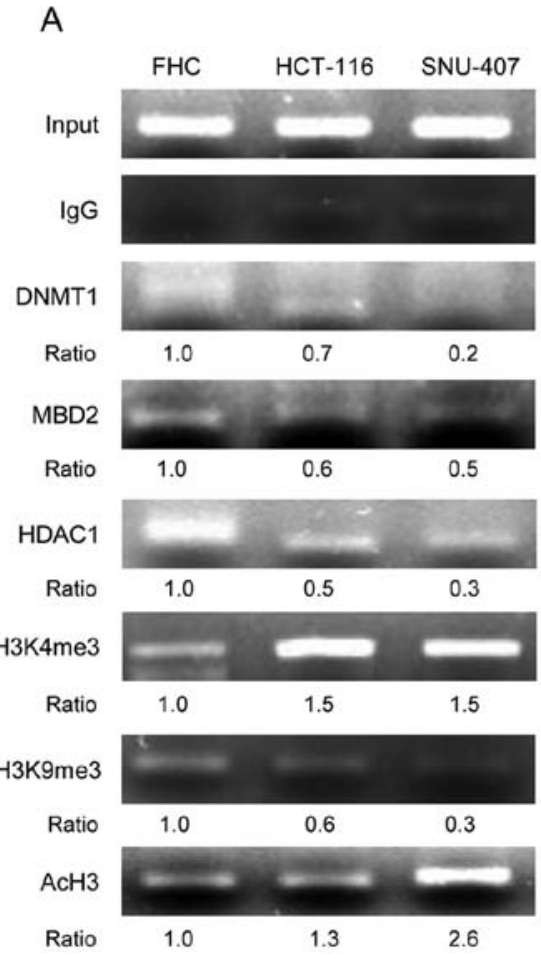

B

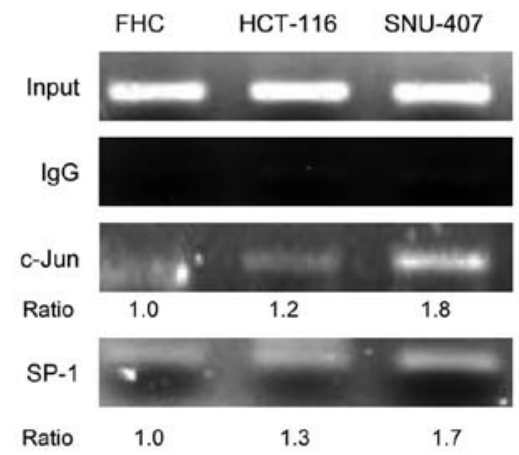

Figure 5. GSTP-1 transcriptional activity correlates with DNA methylation and histone modification. Chromatin from FHC, HCT-116 and SNU-407 cell lines was immunoprecipitated with specific antibodies. Associated DNA was amplified using primers specific for the GSTP-1 promoter region, and PCR products were separated on an agarose gel stained with RedSafe ${ }^{\mathrm{TM}}$ nucleic acid staining solution. (A) Levels of DNMT1, MBD2, HDAC1, H3K4me3, $\mathrm{H} 3 \mathrm{~K} 9 \mathrm{me} 3$ and $\mathrm{AcH} 3$ in the GSTP-1 promoter region were quantified, and are presented as an index of intensity. (B) The association of c-Jun and SP-1 with the GSTP-1 promoter was detected and quantified. Input represents amplification of total DNA from a whole cell lysate.

associated with the GSTP-1 promoter region in the HCT-116 and SNU-407 cell lines (Fig. 5B). These data reveal that the upregulation of GSTP-1 in human colorectal cancers is probably due to reduced methylation of the GSTP-1 promoter and active form of histone modification and resulting in the increased activity of specific transcription factors.

Depletion of GSTP-1 induces the sensitivity of colorectal cancer cell lines to anticancer agent. To investigate whether GSTP-1 is involved in drug resistance, SNU-407 cells transfected with GSTP-1 siRNA were treated with the anticancer agent 5-FU, at the $\mathrm{IC}_{50}$ value of $800 \mu \mathrm{M}$, for $48 \mathrm{~h}$. siRNA- 


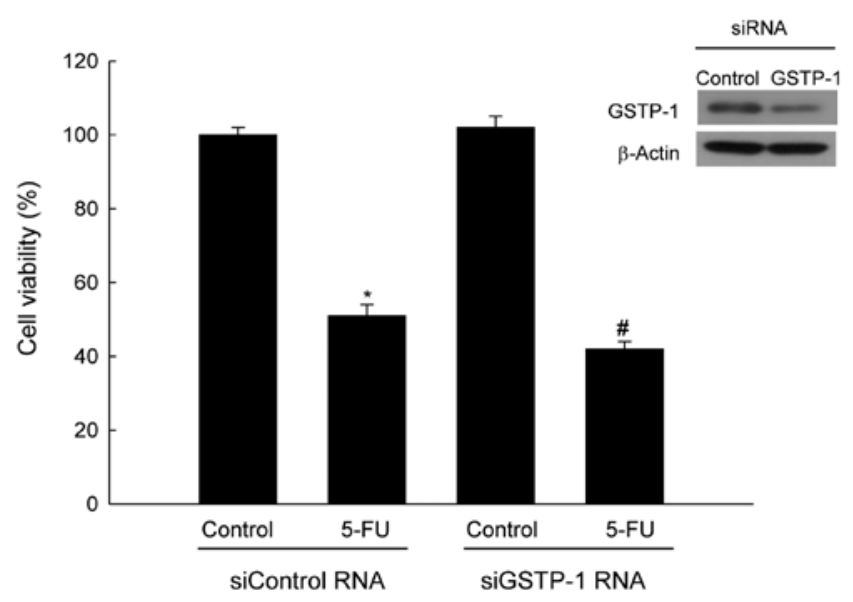

Figure 6. Effect of depletion of GSTP-1 on sensitivity to 5-fluorouracil in SNU-407 cells. SNU-407 cells were transfected with GSTP-1 siRNA or control siRNA. Cell viability was measured after 48-h treatment with 5-FU using the MTT assay. *Significantly different from siControl cells $(\mathrm{P}<0.05)$; ${ }^{\#}$ significantly different from 5-FU-treated siControl cells $(\mathrm{P}<0.05)$.

mediated suppression of GSTP-1 expression increased the sensitivity of SNU-407 cells to 5-FU (Fig. 6). These results indicate that knockdown of GSTP-1 in SNU-407 cells increases sensitivity to the anticancer drug.

\section{Discussion}

GSTP-1 is a phase-II detoxifying enzyme that catalyzes the nucleophilic attack of glutathione on electrophilic compounds (34). Recently, there has been considerable clinical interest in GSTP-1 as a tumor marker and therapeutic target. Overexpression of GSTP-1 in cancer cells is associated with increased resistance to anticancer agents (35). Furthermore, a number of studies have shown that GSTP-1 is the predominant GST isozyme found in human cancer.

In this study, we examined GSTP-1 protein expression and activity in normal and tumor specimens obtained from Korean colon cancer patients. GSTP-1 protein expression and enzyme activity were higher in colon cancer tissues than in the surrounding normal tissues, and overexpression of GSTP-1 in colon cancer tissues was confirmed by histological analysis. This pattern was also observed in colon cancer cell lines. To explore the precise mechanism underlying the upregulation of GSTP-1 in human colon cancers, we investigated GSTP-1 promoter methylation status and the contributions of transcriptional regulators such as DNA methylation, histone modifications and promoter-associated proteins to GSTP-1 expression levels. Promoter methylation status and GSTP-1 expression patterns somewhat correlated in colon cancer cell lines. However, the relationship between promoter methylation status and the pattern of GSTP-1 expression in colon tissues from patients remains speculative. Therefore, we hypothesized that GSTP-1 expression is not regulated exclusively by DNA methylation, but is also synergistically regulated by histone modifications, changes in promoter-associated proteins and recruitment of transcription factors. Indeed, the levels of DNA methylation- and heterochromatin-associated proteins were reduced in human colorectal cell lines in this study. Furthermore, the level of the repressive histone modification $\mathrm{H} 3 \mathrm{~K} 9$ me 3 was reduced in human colorectal cells. In addition, the levels of the permissive histone modification $\mathrm{H} 3 \mathrm{~K} 4 \mathrm{me} 3$ and acetylation of histone $\mathrm{H} 3$ were enriched in human colorectal cells.

The transcriptional regulation of GST proteins of the $\pi$ class has been well characterized in the context of rodent GSTP-1 $(36,37)$. The upstream region of the GSTP-1 gene contains a TPA response element and a related enhancer element termed GSTP-1 enhancer 1, which has characteristics similar to those of antioxidant response elements and electrophile response elements. GSTP-1 enhancer 1 is a specific enhancer of the rat GST $\pi$ gene, but is not present in the $\pi$ class GST genes of mouse or human or in genes of the other GST isoenzymes (38). Therefore, we speculated that the regulatory mechanisms of the human GSTP-1 gene expression might differ from those of rodents. The analysis of the human GSTP-1 proximal promoter in human leukemia has revealed the presence of two putative SP-1 binding sites, located from -57 to -49 and from -47 to -39 , and an AP-1 response element from -69 to -63 $(28,39)$. We detected an association between these transcription factors and the GSTP-1 promoter region. Binding of SP-1 and AP-1 to the GSTP-1 promoter was markedly enhanced in human colorectal cancer cells. Finally, knockdown of GSTP-1 reduced cell proliferation and increased sensitivity to chemotherapy. These observations suggest that GSTP-1 might be a survival factor for colon carcinoma cells. In summary, this study indicates that GSTP-1 may serve as a clinically useful biomarker of colon cancer and as a target for anticancer drugs.

\section{Acknowledgements}

This study was supported by a grant from the National R\&D Program for Cancer Control, Ministry for Health and Welfare, Korea (1120340).

\section{References}

1. Coles BF, Chen G, Kadlubar FF and Radominska-Pandya A: Interindividual variation and organ-specific patterns of glutathione $\mathrm{S}$ transferase alpha, mu, and pi expression in gastrointestinal ltract mucosa of normal individuals. Arch Biochem Biophys 403: 270-276, 2002.

2. Hayes JD, Flanagan JU and Jowsey IR: Glutathionetransferases. Annu Rev Pharmacol Toxicol 45: 51-88, 2005.

3. Board PG, Webb GC and Coggan M: Isolation of a cDNA clone and localization of the human glutathione S-transferase 3 genes to chromosome bands 11q13 and12q13-14. Ann Hum Genet 53: 205-213, 1989.

4. Islam MQ, Platz A, Szpirer J, Szpirer C, et al: Chromosomal localization of human glutathione transferase genes of classes alpha, mu, and pi. Hum Genet 82: 338-342, 1989.

5. Ranganathan $\mathrm{S}$ and Tew KD: Immunohistochemical localization of glutathione S-transferases $\alpha, \mu$, and $\pi$ in normal tissue and carcinomas from human colon. Carcinogenesis 12: 2383-2387, 1991.

6. Miyanishi K, Takayama T, Ohi M, et al: Glutathione Stransferase- $\pi$ overexpression is closely associated with $\mathrm{K}$-ras mutation during human colon carcinogenesis. Gastroenterology 121: 865-874, 2001.

7. Adler V, Yin Z, Fuchs SY, et al: Regulation of JNK signaling by GSTp. EMBO J 18: 1321-1334, 1999.

8. Townsend DM, Manevich Y, He L, Hutchens S, et al: Novel role for glutathione S-transferase pi: regulator of protein S-glutathionylation following oxidative and nitrosative stress. J Bio Chem 284: 436-445, 2009.

9. Lo HW and Ali-Osman F: Genetic polymorphism and function of glutathione S-transferases in tumor drug resistance. Curr Opin Pharmacol 7: 367-374, 2007. 
10. Henderson CJ, Smith AG, Ure J, Brown K, et al: Increased skin tumorigenesis in mice lacking pi class glutathione $\mathrm{S}$-transferases. Proc Natl Acad Sci USA 95: 5275-5280, 1998.

11. Wright JL and Lange PH: Newer potential biomarkers in prostate cancer. Rev Urol 9: 207-213, 2007.

12. Chen CL, Sheen TS, Lou LU and Huang AC: Expression of multidrug resistance 1 and glutathione-S-transferase-Pi protein in nasopharyngeal carcinoma. Hum Path 32: 1240-1244, 2001.

13. Cullen KJ, Newkirk KA, Schumaker LM, Aldosari N, et al: Glutathione-S-transferase pi amplification is associated with cisplatin resistance in head and neck squamous cell carcinoma cell lines and primary tumors. Cancer Res 63: 8097-8102, 2003.

14. Li M and $\mathrm{Gu}$ J: Changing patterns of colorectal cancer in China over a period of 20 years. World J Gastroenterol 11: 4685-4688, 2005.

15. Kinzler KW and Vogelstein B: Lessons from hereditary colon cancer. Cell 87: 159-170, 1996.

16. Jones PA and Baylin SB: The fundamental role of epigenetic events in cancer. Nat Rev Genet 3: 415-428, 2002.

17. Cheung P, Allis CD and Sassone-Corsi P: Signaling to chromatin through histone modifications. Cell 103: 263-271, 2000.

18. Wang Y, Wysocka J, Sayegh J, et al: Human PAD4 regulates histone arginine methylation levels via demethylimination. Science 306: 279-283, 2004.

19. Garinis GA, Patrinos GP, Spanakis NE and Menounos PG: DNA hypermethylation: when tumour suppressor genes go silent. Hum Gene 111: 115-127, 2002

20. Craig JM: Heterochromatin - many flavours, common themes. Bioessays 27: 17-28, 2005.

21. Robertson KD and Wolffe AP: DNA methylation in health and disease. Nat Rev Gene 1: 11-19, 2000.

22. Heard E, Rougeulle C, Arnaud D, Avner P, et al: Methylation of histone $\mathrm{H} 3$ at Lys-9 is an early mark on the $\mathrm{X}$ chromosome during X inactivation. Cell 107: 727-738, 2001.

23. Rountree MR, Bachman KE, Herman JG and Baylin SB: DNA methylation, chromatin inheritance, and cancer. Oncogene 20 3156-3165, 2001

24. Watt PM, Kumar R and Kees UR: Promoter demethylation accompanies reactivation of the HOX11 proto-oncogene in leukemia. Genes Chromosomes Cancer 29: 371-377, 2000.

25. Schübeler D, MacAlpine DM, Scalzo D, et al: The histone modification pattern of active genes revealed through genomewide chromatin analysis of a higher eukaryote. Genes Dev 18 1263-1271, 2004.

26. Berger SL: The complex language of chromatin regulation during transcription. Nature 447: 407-412, 2007.
27. Moffat GJ, McLaren AW and Wolf CR: Sp1-mediated transcriptional activation of the human Pi class glutathione S-transferase promoter. J Bio Chem 271: 1054-1060, 1996.

28. Duvoix A, Schnekenburger M, Delhalle S, et al: Expression of glutathione S-transferase P1-1 in leukemic cells is regulated by inducible AP-1 binding. Cancer Lett 216: 207-219, 2004.

29. Morceau F, Duvoix A, Dehalle S, Schnekenburger M, et al: Regulation of glutathione S-transferase P1-1 gene expression by NF-kappa B in tumor necrosis factor alpha-treated K562 leukemia cells. Biochem Pharma 67: 1227-1238, 2004.

30. Moon PG, Lee JE, You S, et al: Proteomic analysis of urinary exosomes from patients of early $\operatorname{IgA}$ nephropathy and thin basement membrane nephropathy. Proteomics 11: 2459-2475, 2011.

31. Silva JC, Denny R, Dorschel CA, et al: Quantitative proteomic analysis by accurate mass retention time pairs. Anal Chem 77: 2187-2200, 2005.

32. Karius T, Schnekenburger M, Ghelfi J, Walter J, et al: Reversible epigenetic fingerprint-mediated glutathione-S-transferase P1 gene silencing in human leukemia cell lines. Biochem Pharma 81: 1329-1342, 2011

33. Carmichael J, DeGraff WG, Gazdar AF, Minna JD, et al: Evaluation of a tetrazolium-based semiautomated colorimetric assay: assessment of chemosensitivity testing. Cancer Res 47: 936-941, 1987.

34. Rushmore TH and Pickett CB: Glutathione S-transferases, structure, regulation, and therapeutic implications. J Bio Chem 268: 11475-11478, 1993

35. Townsend DM and Tew KD: The role of glutathione-S-transferase in anti-cancer drug resistance. Oncogene 22: 7369-7375, 2003.

36. Sakai M, Okuda A and Muramatsu M: Multiple regulatory elements and phorbol 12-O-tetradecanoate-13-acetate responsiveness of the rat placental glutathione transferase gene. Proc Nat Acad Sci USA 85: 9456-9460, 1988

37. Osada S, Takano K, Nishihara T, Suzuki $\mathrm{T}$, et al: CCAAT/enhancer-binding proteins alpha and beta interact with the silencer element in the promoter of glutathione S-transferase $\mathrm{P}$ gene during hepato-carcinogenesis. J Bio Chem 270: 31288-31293, 1995.

38. Dixon KH, Cowell IG, Xia CL, Pemble SE, et al: Control of expression of the human glutathione $\mathrm{S}$-transferase pi gene differs from its rat orthologue. Biochem Biophy Res Commun 163: 815-822, 1989

39. Duvoix A, Schmitz M, Schnekenburger M, et al: Transcriptional regulation of glutathione S-transferase P1-1 in human leukemia. Biofactors 17: 131-138, 2003 